\title{
Aplicación del modelo SCOR en Pymes metalmecánicas de Olavarría
}

\section{Application of the SCOR model in metalworking SMEs from Olavarría}

DOI: http://dx.doi.org/10.17981/ingecuc.12.2.2016.05

Artículo de Investigación Científica - Fecha de Recepción: 16 de Mayo de 2016, Fecha de Aceptación: 30 de Agosto de 2016

María Emilia Spina

Facultad de Ingeniería, Universidad Nacional del Centro de la Provincia de Buenos Aires Olavarría, Argentina. spinaemilia@gmail.com

Claudia Rohvein*

Facultad de Ingeniería, Universidad Nacional del Centro de la Provincia de Buenos Aires

Olavarría, Argentina. crohvein@fio.unicen.edu.ar

Silvia Urrutia

Facultad de Ingeniería, Universidad Nacional del Centro de la Provincia de Buenos Aires

Olavarría, Argentina. surrutia@fio.unicen.edu.ar

Geraldina Roark

Facultad de Ingeniería, Universidad Nacional del Centro de la Provincia de Buenos Aires

Olavarría, Argentina. groark@fio.unicen.edu.ar

Diana Paravié

Facultad de Ingeniería, Universidad Nacional del Centro de la Provincia de Buenos Aires

Olavarría, Argentina. dparavie@fio.unicen.edu.ar

\section{Guillermo Corres}

Facultad de Ciencias Económicas, Universidad Nacional del Centro de la Provincia de Buenos Aires

Tandil, Argentina. corres@rec.unicen.edu.ar

Cómo citar este artículo:

M.E. Spina, C. Rohvein, S.Urrutia, G. Roark, D. Paravié y G. Corres "Aplicación del modelo SCOR en PyMEs metalmecánicas de Olavarría”, INGE CUC, vol. 12, No.2, pp. 50-57, 2016. DOI: http://dx.doi.org/10.17981/ingecuc.12.2.2016.05

Resumen- Es conocido el aporte de las Pymes en la economía del país así como sus problemas en gestión. Una solución la brinda la medición del desempeño, la cual permite conocer cuantitativamente su comportamiento. Para esto el modelo de referencia de operaciones (SCOR) diseñado por el Supply Chain Council es de gran ayuda. La metodología de investigación de este trabajo muestra un enfoque mixto con un método no experimental, cuyo diseño es transeccional descriptivo. El objetivo consiste en definir las distintas partes que conformarán el modelo SCOR para Pymes metalmecánicas de Olavarría (Argentina) de modo de identificar la cadena de suministro y la categoría de cada proceso, como así también definir los indicadores y las mejores prácticas para cada configuración. El sector se dividió en cuatro subgrupos y se observó que todos trabajan con productos a diseño salvo fabricación de aberturas de aluminio que, además, realiza productos a pedido y para stock.

Palabras clave- cadena de suministro, SCOR; medición de desempeño, pymes industriales.

\begin{abstract}
It is known the contribution of SMEs in the economy as well as its management problems. One solution is the use of performance metrics in order to quantitatively understand their behavior. For this purpose, is really helpful the operations reference model (SCOR) designed by the Supply Chain Council. The research methodology of this paper presents a mixed approach with a non-experimental method, whose design is descriptive transversal. The aim is to define the different parts that make up the SCOR model for metalworking SMEs from Olavarria, Argentina, in order to identify the supply chain and category of each process, as well as define indicators and best practices for each configuration. The sector has been divided into four subgroups and it was found that all of them work with design products, except of the manufacturing of aluminum apertures that also makes customized products and stock products.
\end{abstract}

Keywords- supply chain, SCOR, performance measurement, SMEs.

"Autor de Correspondencia: crohvein@fio.unicen.edu.ar 


\section{INTRODUCCIÓN}

Hoy en día, dado el entorno cada vez más complejo, se piensa que las organizaciones no solo deben mejorar su desempeño interno, sino que también deben considerar la integración, tanto con proveedores como con clientes. Precisamente esto es una oportunidad de trabajar sobre la cadena de suministro (CS) para mejorar su planificación y beneficios a largo plazo [1], [2].

Muchas CS carecen de un desempeño adecuado debido, entre otros aspectos, a la falta de integración y coordinación en sus procesos, ausencia de técnicas de gestión logística que faciliten su diseño y operación, obviando integraciones necesarias entre sus elementos. Además, no se encuentran definidos los objetivos estratégicos de la organización a través de los procesos en la CS, por lo que se desconoce su contribución al rumbo estratégico de la entidad [3].

Para conocer cuantitativamente el comportamiento de la CS la medición es fundamental. En [4] reconocen que un punto crítico en la evaluación del desempeño de una compañía y de su CS es la elección de indicadores apropiados para cada caso, ya que de su análisis se detectarán las áreas factibles de mejora que les permitan tener éxito competitivo.

No obstante, en [3] se reporta que la falta de indicadores en las organizaciones hace que no se cuente con la información apropiada para la toma de decisiones, lo cual refleja en una mala gestión, elevación de costos y deficiencias en el servicio brindado.

De este modo, el buen desempeño de la totalidad de la CS es fundamental para cualquier industria, por lo que es necesario buscar enfoques innovadores que beneficien conjuntamente a todos sus actores.

$\mathrm{Al}$ ser un tema que cada día gana mayor importancia en la gestión empresarial, existen varios modelos para evaluar y administrar eficientemente la denominada CS. Una acertada opción es el modelo de referencia de operaciones de la cadena de suministro (SCOR) diseñado por el Supply Chain Council. El mismo es una herramienta estratégica que presenta ventajas como terminología unificada, procesos estandarizados, indicadores de desempeño asociados a cada proceso y flexibilidad para adaptarse a cada organización [5].

Por otro lado, las pequeñas y medianas empresas (pymes) son agentes importantes en la estructura económica de los países, no solo por su participación en la cantidad de firmas sino por su aporte en la agregación de valor y en la generación de empleo [6][9].

A pesar de su importante papel en el desarrollo del país ha sido relevado por organismos gubernamentales, que estas empresas presentan diversos problemas asociados esencialmente a la informalidad de su gestión, con el consecuente incremento de costos productivos y la disminución de rentabilidad [10], [11].
De igual modo, la industria de la ciudad de Olavarría no es ajena a esos problemas, tal como afirma [12], quien reportó que los principales problemas son: aumento de los costos directos de producción, fuerte competencia, caída de las ventas, dificultades en la obtención de financiamiento e insuficiente capacidad instalada.

La gestión realizada por las pymes de Olavarría es una gestión reactiva, orientada hacia el interior, que no utiliza herramientas de planificación o de evaluación, sus líderes se involucran tanto en las labores cotidianas que no les queda tiempo para las planificaciones y decisiones estratégicas [13], [14].

Sin embargo, en [15] se destaca que la mayor contribución al PBI en la región lo origina la industria olavarriense. Este dato refuerza la relevancia de aportar para contrarrestar las dificultades a las que se enfrentan.

En pos de delimitar el alcance, este trabajo es una contribución para el sector metalmecánico de la ciudad de Olavarría, el cual es considerado estratégico para el desarrollo de la ciudad por ser un eslabón fundamental en la articulación con otros sectores industriales [15], [16].

En función de la situación descripta en las pymes se piensa que un buen comienzo para revertir las debilidades es promover el uso de herramientas prácticas para la evaluación e implementar procesos de mejora de la gestión de la CS. Para ello se hace indispensable el uso de una serie de parámetros cuantificables que permitan analizarla y gestionarla de una forma objetiva.

De esta manera se aportaría a las pymes de referencia una herramienta para identificar y mejorar el desempeño de la CS mediante el uso de indicadores. Además de contar con la información para tomar las decisiones que permitan el despliegue adecuado del rumbo estratégico de la organización y la toma de decisiones efectivas y oportunas.

En este sentido, el objetivo de este trabajo es definir las distintas partes que conformaran el modelo SCOR para pymes metalmecánicas de Olavarría, con el propósito de identificar la cadena de suministro y la categoría de cada proceso, y también definir los indicadores y las mejores prácticas para cada configuración.

\section{MÉTodo}

El proyecto en el que se enmarca este trabajo presenta un enfoque de investigación mixto, ya que combina componentes cuantitativos y cualitativos, con preponderancia del método cuantitativo.

$\mathrm{Su}$ diseño es explicativo secuencial, en el cual se recaban y analizan datos cuantitativos complementados con datos cualitativos. La mezcla mixta ocurre cuando los resultados cuantitativos informan a los datos cualitativos. El método es no experimental, cuyo diseño es transeccional descriptivo. 
Para la selección de la muestra se tomó como referencia la base de datos de ARBA (Agencia de Recaudación de la Provincia de Buenos Aires) suministrada por la Agencia de Desarrollo Local de Olavarría, en la que se identificaron un total de 98 mipymes metalmecánicas en el partido de Olavarría, Provincia de Buenos Aires (Argentina). Seguidamente se limitó la población de estudio a 45 empresas pymes industriales pertenecientes al sector metalmecánico localizadas físicamente en la cuidad de Olavarría y con una trayectoria reconocida. Considerando que se conoce el tamaño poblacional se seleccionó una muestra probabilística de 23 empresas mediante el empleo de un muestro aleatorio simple. Posteriormente, la muestra se estratificó en cuatro subsectores que se corresponden con Fabricación de aberturas de aluminio; Fabricación de carrocerías; Construcción de estructuras metálicas, Prestación de servicios y montaje industrial; Mecanizado. Para asegurar que dichos estratos fueran representativos, se buscó que el porcentaje de pymes en cada estrato de la muestra sea similar al porcentaje del estrato correspondiente en la población.

De este modo se contó con una base de datos 2012-2013 en la que se dispone de características representativas de las pymes de referencia. Las mismas fueron relevadas mediante la aplicación de un instrumento de recolección de datos válido de acuerdo con la metodología descripta en [17]. El mismo contiene preguntas cerradas y abiertas, que contemplan enfoques cualitativos y cuantitativos respecto a las distintas variables por analizar. Los ejes de indagación versan sobre las actividades primarias y de apoyo, integrantes de la cadena de valor, focalizando las preguntas en el tipo de recursos que utilizan cada una de las actividades y su gestión. Los recursos tangibles e intangibles son las variables independientes de investigación, y las variables dependientes son las actividades primarias y de apoyo.

Finalmente se hace uso de la base de datos para aplicar el modelo de referencia SCOR en la muestra objeto de estudio.

\section{Desarrollo}

La gestión de la cadena de suministro no implica mejorar procesos de forma independiente, sino ver todas las empresas como una sola y busca alcanzar un beneficio global. Este concepto incluye tres dimensiones: una dimensión estratégica, en la que es necesario que exista una coordinación de las organizaciones de la CS en pos de lograr una visión sistémica; una dimensión táctica, que implica visualizar los flujos como procesos y entenderlos como secuencias de actividades que cruzan horizontalmente más de una organización; y una dimensión operativa, que involucra tareas operacionales como almacenar, producir o distribuir, realizadas en for- ma interna en coordinación con otras organizaciones o mediante otras organizaciones externas.

El modelo de referencia SCOR proporciona un marco único que vincula los procesos de negocio, las métricas, las mejores prácticas y la tecnología en una estructura unificada para apoyar la comunicación entre los socios de la CS y mejorar la eficacia de la gestión de la misma.

Este modelo se organiza en torno a los cinco procesos de gestión primaria: Planificación (P), Abastecimiento (S), Producción (M), Distribución (D) y Retorno (R), abarcando las interacciones con los proveedores y clientes, las transacciones de material físico y las interacciones de mercado.

En cuanto al desempeño de la CS, SCOR trabaja con dos tipos de elementos: los atributos de desempeño y las métricas. Los primeros son utilizados para expresar una estrategia y SCOR identifica cinco atributos de la CS: fiabilidad, capacidad de respuesta, agilidad, costos y gestión de activos. Las métricas proporcionan la base para medir el éxito en el logro de los objetivos deseados.

En cuanto a las definiciones de los atributos, "fiabilidad" se refiere a la capacidad para realizar tareas como se esperaba; "capacidad de respuesta" describe la velocidad a la que se realizan las tareas; "agilidad" representa la capacidad de responder a las influencias externas y a la capacidad de cambiar; "costos" refiere el costo de operación del proceso, y "gestión de activos" describe la capacidad de utilizar de manera eficiente los activos.

Para poner en funcionamiento el modelo SCOR es fundamental definir cada uno de los elementos que formarán parte del mismo. Esto consiste en identificar la cadena de suministro y la categoría de cada proceso, definir los indicadores y las mejores prácticas para cada configuración.

\section{A. Identificación de la cadena de suministro}

En primera instancia se define el alcance que tendrá el modelo con respecto a la cadena de suministro.

En las figuras 1, 2, 3 y 4 se muestran las cadenas de suministro para cada subsector industrial estudiado, refiriendo los principales proveedores y clientes.

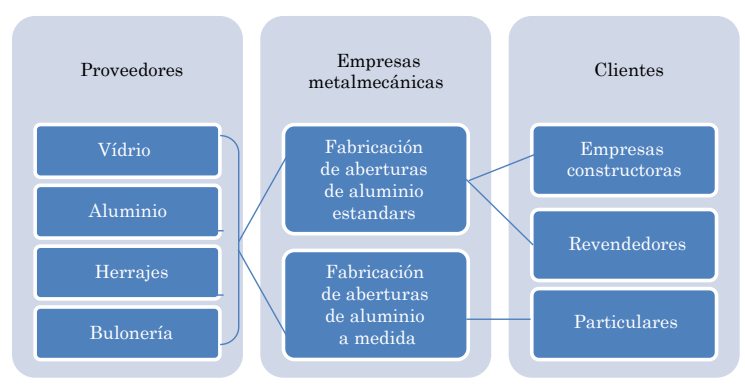

Fig. 1. Cadena de suministro de empresas de fabricación de aberturas de aluminio. Fuente: Elaboración propia. 


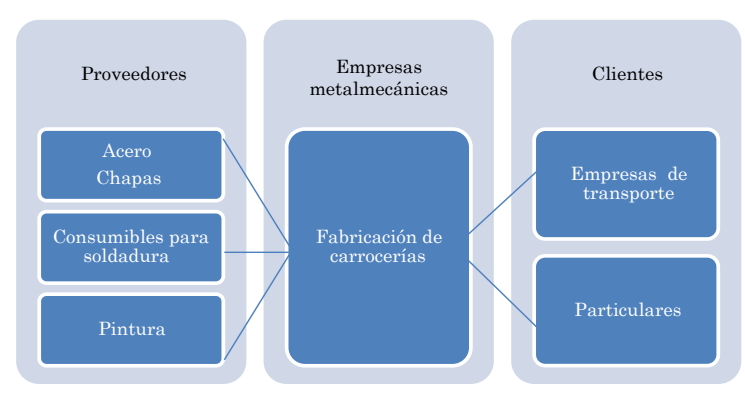

Fig. 2. Cadena de suministro de empresas de fabricación de carrocerías. Fuente: Elaboración propia.

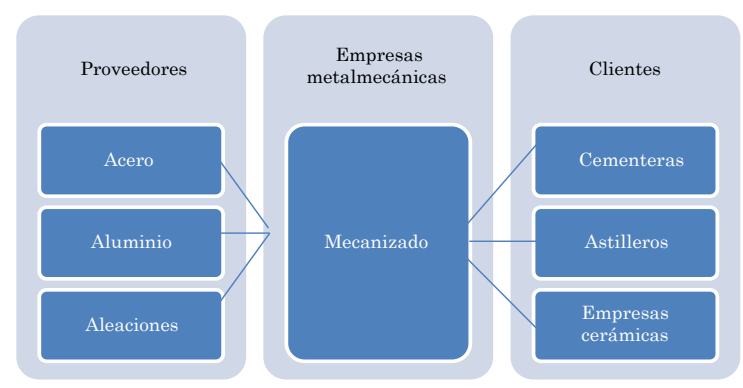

Fig. 3. Cadena de suministro de empresas de fabricación de mecanizado. Fuente: Elaboración propia.

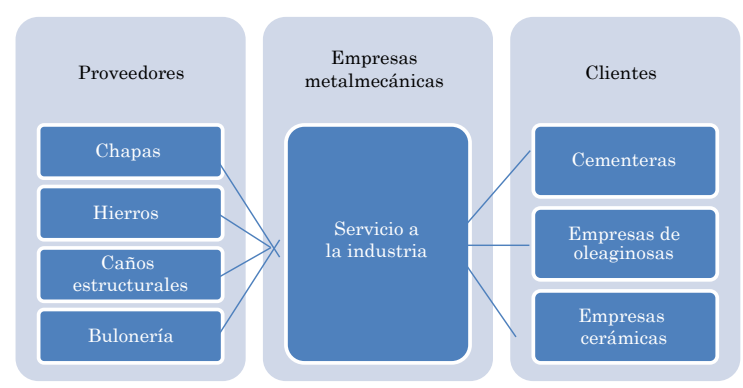

Fig. 4. Cadena de suministro de empresas que prestan servicio a la industria. Fuente: Elaboración propia.

\section{B. Identificación de la categoría de procesos}

Dado que cada una de las métricas debe ir asociada a un proceso, es fundamental definir los que realmente se realizan en las pymes objeto de estudio. Para ello se genera un esquema con los procesos básicos de nivel 1 (tipos de procesos) y 2 (categoría de procesos) vinculado a cada uno de los subsectores industriales relevados.

El nivel 1 o nivel superior contiene cinco procesos básicos de gestión. El primero, planificación, corresponde al plan de acción que tiene por objetivo balancear los recursos con los requerimientos de la empresa para cubrir la demanda en un horizonte de tiempo más largo. Abastecimiento o compra de los bienes o servicios es el proceso que permite cumplir lo estipulado en la planeación. Comprende la adquisición, entrega, recibo y transferencia de las materias primas, productos sub ensamblados, productos terminados y servicios. Incluye ingeniería para órdenes de producto, inventarios y cumplimiento de órdenes. La producción comprende la elaboración y/o transformación de las materias primas hasta su estado final. En la distribución se entregan los bienes o servicios para satisfacer la demanda y cumplir el plan. Finalmente, los retornos son los procesos para devolver un material considerado defectuoso, los mismos pueden ser desde los clientes hacia la organización o desde la organización hacia los proveedores.

El nivel 2, o nivel de configuración, considera las diferentes categorías de procesos que se visualizan en la tabla 1 .

Tabla 1. Descripción de los dos niveles de proceso

\begin{tabular}{|c|c|}
\hline Nivel 1 & Nivel 2 \\
\hline \multirow{5}{*}{ Planear $(\mathrm{P})$} & Planificación de la cadena de suministro (P1) \\
\hline & Planificación del abastecimiento (P2) \\
\hline & Planificación de la producción (P3) \\
\hline & Planificación de la distribución (P4) \\
\hline & Planificación del retorno (P5) \\
\hline \multirow{3}{*}{$\begin{array}{l}\text { Abasteci- } \\
\text { miento (S) }\end{array}$} & Abastecer para stock (S1) \\
\hline & Abastecer para pedido (S2) \\
\hline & Abastecer productos a diseño (S3) \\
\hline \multirow{3}{*}{$\begin{array}{l}\text { Producción } \\
\text { (M) }\end{array}$} & Producción para stock (M1) \\
\hline & Producción para pedido (M2) \\
\hline & Producción productos a diseño (M3) \\
\hline \multirow{4}{*}{$\begin{array}{l}\text { Distribución } \\
\text { (D) }\end{array}$} & Distribución de producto en stock (D1) \\
\hline & Distribución a pedido (D2) \\
\hline & Distribución productos a diseño (D3) \\
\hline & Distribución productos a minoristas (D4) \\
\hline \multirow{3}{*}{$\begin{array}{l}\text { Retorno a } \\
\text { proveedor } \\
\text { (SR) }\end{array}$} & Retorno de productos defectuosos (SR1) \\
\hline & $\begin{array}{l}\text { Retorno productos para mantenimiento, repa- } \\
\text { ración o reacondicionamiento (SR2) }\end{array}$ \\
\hline & Retorno de productos en exceso (SR3) \\
\hline \multirow{3}{*}{$\begin{array}{l}\text { Retorno de } \\
\text { cliente (DR) }\end{array}$} & Retorno de productos defectuosos (DR1) \\
\hline & $\begin{array}{l}\text { Retorno productos para mantenimiento, repa- } \\
\text { ración o reacondicionamiento (DR2) }\end{array}$ \\
\hline & Retorno de productos en exceso (DR3) \\
\hline
\end{tabular}

Fuente: [5].

En el nivel 3, o nivel de elementos de procesos, se descomponen los procesos en sus elementos constituyentes, se definen las métricas y las mejores prácticas. El detalle correspondiente a este nivel no se abarca debido a la extensión de su tratamiento.

Al abordar el análisis de los procesos de nivel 1 y 2 no se profundiza en el proceso de planificación debido a que su tratamiento no agrega valor al trabajar con una muestra estratificada, sino que es necesario aplicarlo a cada empresa en particular. 
A continuación se puede observar en las figuras 5 y 6 que el subsector" fabricación de aberturas de aluminio" es el único que presenta la particularidad de trabajar de dos maneras muy diferentes; por un lado, algunas empresas de la muestra realizan productos con medidas estándar, mientras que el resto fabrica productos a medida. En la Fig. 5 "stock" representa toda materia prima o producto terminado almacenado, mientras que "pedido" se corresponde con aquella materia prima requerida para realizar un producto solicitado por el cliente según las medidas estándar ofrecidas por la empresa.

Los subsectores "carrocerías", "mecanizados" y "servicio a la industria" fabrican productos a medida, por lo que su configuración en la categoría de procesos está representada por la Fig. 6.

En la Fig. 6 se observa que en el proceso de abastecimiento, las empresas compran tanto para almacenar materia prima como para cubrir las necesidades específicas de producir un producto a diseño. Cuando se habla de "producto a diseño" se refiere a todo aquel producto que, según el cliente, presente distintas particularidades.

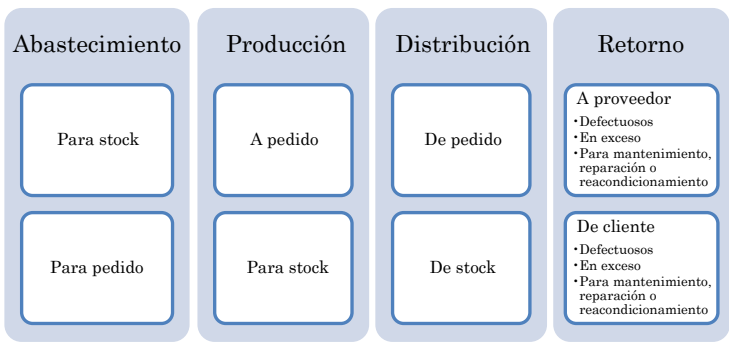

Fig. 5. Categoría de procesos para empresas que realizan productos estándar en fabricación de aberturas de aluminio. Fuente: Elaboración propia.

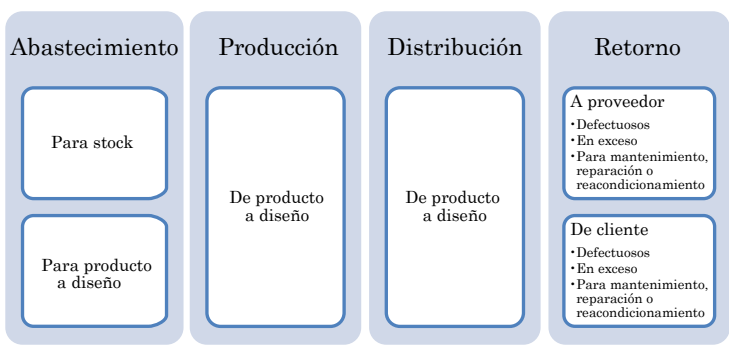

Fig. 6. Categoría de procesos para empresas que realizan productos a medida en fabricación de aberturas de aluminio, en carrocerías, en mecanizados y en empresas que realizan servicio a la industria.

Fuente: Elaboración propia.

\section{Definición de indicadores}

Con el fin de brindar elementos de soporte para recopilar información certera y decidir dónde se debe comenzar a implementar cambios se definen indicadores asociados a los procesos identificados previamente.
El modelo SCOR trabaja con una serie de atributos: fiabilidad, respuesta, agilidad, costos y gestión de activos, que a su vez se subdividen en distintos indicadores, asociados a los tres niveles de procesos.

- Nivel 1: son diagnósticos para la salud general de la cadena de suministro. Estas métricas son también conocidas como indicadores estratégicos y los indicadores clave de rendimiento (KPI). La evaluación comparativa del nivel 1 de métricas ayuda a establecer objetivos realistas que apoyan los objetivos estratégicos.

- Nivel 2: sirven como agentes de diagnóstico para el nivel 1. La relación de diagnóstico ayuda a identificar la causa o causas de una brecha de desempeño para una métrica de nivel 1 .

- Nivel 3: sirven como agentes de diagnóstico para el nivel 2 métricas.

Para su correcta identificación se utiliza la siguiente codificación:

XX.Y.Z

$\mathrm{XX}=$ Atributo de desempeño.

- Fb: Fiabilidad

- Rp: Respuesta

- Ag: Agilidad

- Cs: Costos

- Ac: Gestión de activos

$\mathrm{Y}=$ Nivel de la métrica.

$\mathrm{Z}=$ Número identificativo.

En las tablas 2, 3, 4, 5 y 6 se detallan los indicadores de nivel 1 y 2 asociados al atributo de fiabilidad, respuesta, agilidad, costos y gestión de activos, respectivamente.

No es posible mostrar el detalle relativo a los indicadores del nivel 3 debido a la apertura que se realiza en el nivel 2 de configuración. El mismo contiene 21 categorías de procesos, 5 en Planear, 3 en Aprovisionamiento, 3 en Producción, 4 en Distribución y 6 en Retorno; sumando esto a la ampliación que tiene cada uno en el nivel tres.

Del mismo modo, no es posible exponer el detalle de cálculo de cada indicador, si bien se es consciente de su necesidad. Por tanto, se recomienda recurrir a los documentos aportados por [5].

Tabla 2. Indicadores nivel 1 y 2 ASOCIADOS AL ATRIBUTO DE FIABILIDAD

\begin{tabular}{|c|l|}
\hline Nivel 1 & \multicolumn{1}{|c|}{ Nivel 2 } \\
\hline \multirow{2}{*}{$\begin{array}{c}\text { Fb.1.1 } \\
\text { Cumplimiento } \\
\text { de la orden } \\
\text { perfecta }\end{array}$} & $\begin{array}{l}\text { Fb.2.1\% de los pedidos entregados en su } \\
\text { totalidad }\end{array}$ \\
\cline { 2 - 2 } & $\begin{array}{l}\text { Fb.2.2 Cumplimiento de la entrega al } \\
\text { cliente en fecha }\end{array}$ \\
\cline { 2 - 2 } & Fb.2.3 Exactitud de la documentación \\
\cline { 2 - 3 } & Fb.2.4 Condición perfecta \\
\hline
\end{tabular}

Fuente: Elaboración propia basado en [5]. 
TABla 3. Indicadores Nivel 1 y 2 ASOCIADOS AL ATRIBUTO DE RESPUESTA

\begin{tabular}{|c|c|}
\hline Nivel 1 & Nivel 2 \\
\hline \multirow{4}{*}{$\begin{array}{c}\text { Rp.1.1 } \\
\text { Cumplimiento } \\
\text { del tiempo } \\
\text { de ciclo de la } \\
\text { orden }\end{array}$} & Rp.2.1 Tiempo de ciclo del abastecimiento \\
\hline & Rp.2.2 Tiempo de ciclo de la producción \\
\hline & Rp.2.3 Tiempo de ciclo de la distribución \\
\hline & $\begin{array}{l}\text { Rp.2.4 Tiempo de ciclo de la distribución } \\
\text { minorista }\end{array}$ \\
\hline
\end{tabular}

Fuente: Elaboración propia basado en [5].

Tabla 4. Indicadores nivel 1 y 2 ASOCIADOS AL ATRIBUTO DE AGILIDAD

\begin{tabular}{|c|c|}
\hline Nivel 1 & Nivel 2 \\
\hline \multirow{5}{*}{$\begin{array}{l}\text { Ag.1.1 } \\
\text { Flexibilidad de } \\
\text { la cadena de } \\
\text { suministro }\end{array}$} & Ag.2.1 Flexibilidad del abastecimiento \\
\hline & Ag.2.2 Flexibilidad de la producción \\
\hline & Ag.2.3 Flexibilidad de la distribución \\
\hline & $\begin{array}{l}\text { Ag.2.4 Flexibilidad del retorno a los } \\
\text { proveedores }\end{array}$ \\
\hline & $\begin{array}{l}\text { Ag.2.5 Flexibilidad del retorno de los } \\
\text { clientes }\end{array}$ \\
\hline \multirow{5}{*}{$\begin{array}{c}\text { Ag. } 1.2 \\
\text { Adaptabilidad } \\
\text { de la cadena } \\
\text { de suministro } \\
\text { (hacia arriba) }\end{array}$} & $\begin{array}{l}\text { Ag.2.6 Adaptabilidad del abastecimiento } \\
\text { (hacia arriba) }\end{array}$ \\
\hline & $\begin{array}{l}\text { Ag. } 2.7 \text { Adaptabilidad de la producción } \\
\text { (hacia arriba) }\end{array}$ \\
\hline & $\begin{array}{l}\text { Ag.2.8 Adaptabilidad de la distribución } \\
\text { (hacia arriba) }\end{array}$ \\
\hline & $\begin{array}{l}\text { Ag.2.9 Adaptabilidad del retorno a los } \\
\text { proveedores (hacia arriba) }\end{array}$ \\
\hline & $\begin{array}{l}\text { Ag.2.10 Adaptabilidad del retorno de los } \\
\text { clientes (hacia arriba) }\end{array}$ \\
\hline \multirow{3}{*}{$\begin{array}{c}\text { Ag.1.3 } \\
\text { Adaptabilidad } \\
\text { de la cadena } \\
\text { de suministro } \\
\text { (hacia abajo) }\end{array}$} & $\begin{array}{l}\text { Ag.2.11 Inconveniente de la adaptabilidad } \\
\text { del abastecimiento (hacia abajo) }\end{array}$ \\
\hline & $\begin{array}{l}\text { Ag. } 2.12 \text { Inconveniente de la adaptabilidad } \\
\text { de la producción (hacia abajo) }\end{array}$ \\
\hline & $\begin{array}{l}\text { Ag.2.13 Inconveniente de la adaptabilidad } \\
\text { de la distribución (hacia abajo) }\end{array}$ \\
\hline
\end{tabular}

Fuente: Elaboración propia basado en [5].

Tabla 5. Indicadores nivel 1 y 2 ASOCIADOS AL ATRIBUTO DE COSTOS

\begin{tabular}{|c|l|}
\hline Nivel 1 & \multicolumn{1}{|c|}{ Nivel 2} \\
\hline \multirow{4}{*}{$\begin{array}{c}\text { Cs.1.1 Costo de } \\
\text { la gestión de } \\
\text { la cadena de } \\
\text { suministro }\end{array}$} & Cs.2.1 Costo de planificar \\
\cline { 2 - 2 } \begin{tabular}{c} 
Cs.2.2 Costo de abastecer \\
\cline { 2 - 2 }
\end{tabular} & Cs.2.4 Costo de distribuir \\
\cline { 2 - 2 } & Cs.2.5 Costo del retorno \\
\cline { 2 - 2 } & Cs.2.6 Costo de mitigación \\
\hline $\begin{array}{c}\text { Cs.1.2 Costo de } \\
\text { ventas }\end{array}$ & Cs.2.3 Costo de producir \\
\hline
\end{tabular}

Fuente: Elaboración propia basado en [5].
Tabla 6. Indicadores nivel 1 y 2 asociados al ATRIBUTO DE GESTIÓN DE ACTIVOS

\begin{tabular}{|c|c|}
\hline Nivel 1 & Nivel 2 \\
\hline \multirow{3}{*}{$\begin{array}{c}\text { Ac.1.1 } \\
\text { Tiempo de } \\
\text { ciclo Cash- } \\
\text { to-cash }\end{array}$} & Ac.2.1 Días de ventas pendientes \\
\hline & Ac.2.2 Días de inventario de Suministro \\
\hline & Ac.2.3 Días de pago pendientes \\
\hline \multirow{4}{*}{$\begin{array}{c}\text { Ac. } 1.2 \\
\text { Rendimiento } \\
\text { de los activos } \\
\text { fijos de la } \\
\text { cadena de } \\
\text { suministro }\end{array}$} & Ac.2.4 Ingresos de la Cadena de Suministro \\
\hline & $\begin{array}{l}\text { Cs.1.1 Costo de la gestión de la Cadena de } \\
\text { Suministro }\end{array}$ \\
\hline & Cs.1.2 Costo de las ventas \\
\hline & $\begin{array}{l}\text { Ac. } 2.5 \text { Activos fijos de la Cadena de } \\
\text { Suministro }\end{array}$ \\
\hline \multirow{6}{*}{$\begin{array}{c}\text { Ac. } 1.3 \\
\text { Rendimiento } \\
\text { del capital } \\
\text { de trabajo }\end{array}$} & $\begin{array}{l}\text { Cs.1.1 Costo de la gestión de la Cadena de } \\
\text { suministro }\end{array}$ \\
\hline & Cs.1.2 Costo de las ventas \\
\hline & $\begin{array}{l}\text { Ac. } 2.6 \text { Cuentas por pagar (cuentas por pagar } \\
\text { en circulación) }\end{array}$ \\
\hline & $\begin{array}{l}\text { Ac. } 2.7 \text { Cuentas por cobrar (ventas por } \\
\text { cobrar) }\end{array}$ \\
\hline & Ac.2.8 Inventario \\
\hline & Ac.2.4 Ingresos de la Cadena de Suministro \\
\hline
\end{tabular}

Fuente: Elaboración propia basado en [5].

\section{Definición de las mejores prácticas}

Así como a cada proceso se le pueden asociar indicadores, también el modelo define las mejores prácticas para llevarlo a cabo.

A modo ilustrativo, a continuación se detallan las correspondientes a los procesos de nivel 2 identificados en las empresas del sector metalmecánico (tabla 7).

\section{Conclusiones}

Esta investigación surgió de la necesidad de mejoramiento de las capacidades creadoras de ventajas competitivas en las pymes del sector metalmecánico de la ciudad de Olavarría. Sin embargo, la diversidad de debilidades presente en estas empresas y la variedad de temáticas para solucionarlas hace que la aplicación simultánea sea compleja de llevar a cabo. Por lo cual, aquellos instrumentos que permitan el trato integral y sean flexibles serán los más efectivos. Al buscar herramientas que permitan alcanzar este cometido, se observa que ya no basta con mejorar las operaciones o el desempeño hacia el interior de la empresa, sino que se debe ir más allá. Para lograr esto se deja de focalizar en la organización propiamente dicha y se avanza hacia la cadena de suministro.

$\mathrm{Al}$ momento de seleccionar la herramienta se optó por el modelo de referencia de operaciones de la cadena de suministro (SCOR) diseñado por el Supply Chain Council. El mismo es una herramienta estratégica que presenta múltiples ventajas, destacándose entre ellas: unificación de terminología, estandariza- 
Tabla 7. Mejores prácticas correspondientes a los procesos del nivel 2.

\begin{tabular}{|c|c|c|c|c|c|c|c|c|c|c|}
\hline \multirow{2}{*}{ Mejores Prácticas } & \multicolumn{10}{|c|}{ Procesos de nivel 2} \\
\hline & S1 & S2 & S3 & M1 & M2 & M3 & D1 & D2 & SR3 & DR3 \\
\hline $\begin{array}{l}\text { Acuerdos de servicios conjuntos. Sistemas de planificación de } \\
\text { colaboración }\end{array}$ & $\mathrm{X}$ & $\mathrm{X}$ & $\mathrm{X}$ & & & & & & & \\
\hline Utilizar prácticas de compras "verdes" & $\mathrm{X}$ & $\mathrm{X}$ & $\mathrm{X}$ & & & & & & & \\
\hline Seleccionar a los proveedores con sistema de gestión ambiental & $\mathrm{X}$ & $\mathrm{X}$ & $\mathrm{X}$ & & & & & & & \\
\hline Control estadístico de procesos automatizados & & $\mathrm{X}$ & & & & & & & & \\
\hline Administración del inventario del proveedor & & & & $\mathrm{X}$ & & & & & & \\
\hline $\begin{array}{l}\text { Vincular el desempeño individual a los objetivos de la } \\
\text { organización y de las divisiones }\end{array}$ & & & & $\mathrm{X}$ & $\mathrm{X}$ & & & & & \\
\hline $\begin{array}{l}\text { Comparación de los resultados de rendimiento con las mejores } \\
\text { empresas del sector }\end{array}$ & & & & $\mathrm{X}$ & & & & & & \\
\hline Disponibilidad rápida de empleados & & & & $\mathrm{X}$ & & & & & & \\
\hline Proveer capacitaciones continuas a los trabajadores & & & & $\mathrm{X}$ & $\mathrm{X}$ & & & & & \\
\hline Cargar nivel de producción. Planificación de la capacidad & & & & $\mathrm{X}$ & $\mathrm{X}$ & & & & & \\
\hline $\begin{array}{l}\text { Organización para mejorar la flexibilidad. Pocas clasificaciones } \\
\text { de trabajo, fuerza de trabajo autodirigida, estructura de } \\
\text { gestión plana, equipos de trabajo de funciones cruzadas }\end{array}$ & & & & $\mathrm{X}$ & $\mathrm{X}$ & $\mathrm{X}$ & & & & \\
\hline Manufactura dividida en células de trabajo & & & & $\mathrm{X}$ & $\mathrm{X}$ & $\mathrm{X}$ & & & & \\
\hline $\begin{array}{l}\text { Plan preciso y aprobado de las instrucciones de trabajo/ } \\
\text { procesos }\end{array}$ & & & & $\mathrm{X}$ & $\mathrm{X}$ & & & & & \\
\hline $\begin{array}{l}\text { Utilizar un equipo basado en enfoque sistemático para la } \\
\text { identificación y eliminación de desperdicio, o actividades que no } \\
\text { añaden valor dentro de la organización de producción }\end{array}$ & & & & $\mathrm{X}$ & & & & & & \\
\hline $\begin{array}{l}\text { Seguimiento de pedidos sin papel y visibilidad de las órdenes } \\
\text { de los clientes }\end{array}$ & & & & $\mathrm{X}$ & $\mathrm{X}$ & $\mathrm{X}$ & & & & \\
\hline Publicar resultados de rendimientos & & & & $\mathrm{X}$ & $\mathrm{X}$ & $\mathrm{X}$ & & & & \\
\hline Orden de producción sin papeles y seguimiento de inventario & & & & $\mathrm{X}$ & & & & & & \\
\hline $\begin{array}{l}\text { Aplazamiento. El aplazamiento (diferenciación retardada) es } \\
\text { un concepto de la cadena de suministro cuando un producto } \\
\text { se mantiene el mayor tiempo posible en un estado genérico. } \\
\text { La diferenciación del producto genérico en un producto final } \\
\text { específico se desplaza más cerca del consumidor, posponiendo } \\
\text { identificar los cambios, tales como el montaje o embalaje, a la } \\
\text { última ubicación posible cadena de suministro }\end{array}$ & & & & $\mathrm{X}$ & $\mathrm{X}$ & & $\mathrm{X}$ & $\mathrm{X}$ & & \\
\hline $\begin{array}{l}\text { Registros precisos y de bajo costo para la garantía y } \\
\text { seguimiento de la regulación }\end{array}$ & & & & $\mathrm{X}$ & & & & & & \\
\hline Producir productos a necesidades únicas del cliente & & & & & $\mathrm{X}$ & & & & & \\
\hline $\begin{array}{l}\text { Registros precisos y de bajo costo para la garantía y } \\
\text { seguimiento de la regulación }\end{array}$ & & & & & $\mathrm{X}$ & & & & & \\
\hline $\begin{array}{l}\text { Los plazos de entrega se desarrollaron en colaboración con los } \\
\text { clientes }\end{array}$ & & & & & $\mathrm{X}$ & $\mathrm{X}$ & & & & \\
\hline $\begin{array}{l}\text { Diseño del producto en colaboración con el cliente. } \\
\text { Herramientas de diseño en línea }\end{array}$ & & & & & & $\mathrm{X}$ & & & & \\
\hline Pedidos en línea & & & & & & & $\mathrm{X}$ & & & \\
\hline Catálogos comerciales electrónicos & & & & & & & $\mathrm{X}$ & & & \\
\hline Reposición rápida & & & & & & & $\mathrm{X}$ & & & \\
\hline Respuesta rápida y eficiente al consumidor & & & & & & & $\mathrm{X}$ & & & \\
\hline $\begin{array}{l}\text { Habilitar el envío directo al cliente. Activar envíos directos } \\
\text { entre los clientes para reducir el transporte y el manejo general }\end{array}$ & & & & & & & & & $\mathrm{X}$ & $\mathrm{X}$ \\
\hline
\end{tabular}

Fuente: Elaboración propia basado en [5].

Abastecer para stock (S1). Abastecer para pedido (S2). Abastecer para producto a diseño (S3). Producir para stock

(M1). Producir para pedido (M2). Producir producto a diseño (M3). Distribución de stock (D1). Distribución de pedido (D2). Retorno de productos en exceso al proveedor (SR3). Retorno de productos en exceso del cliente (DR3). 
ción de los procesos, logro de una visión global, ya que abarca toda la cadena de suministro, implementación de indicadores de desempeño asociados a cada proceso para determinar el estado de situación actual, análisis desde los aspectos más generales a los más particulares, flexibilidad para adaptarse a cada organización, identificación de procesos por mejorar, definición de las mejores prácticas que se pueden implementar, priorización de los proyectos de mejora de acuerdo con las capacidades que son generadoras de ventajas competitivas.

Como primer paso para adaptar este modelo a las empresas del sector metalmecánico de la ciudad de Olavarría se debió identificar la cadena de suministro. Al ser tan amplias y variadas las actividades dentro del sector, esta resultó poco representativa. Por lo que se concluyó que para un análisis más específico era necesario dividir el sector en subgrupos; con lo cual se logró identificar cuatro cadenas de suministro particulares que se corresponden con Fabricación de aberturas de aluminio; Fabricación de carrocerías; Construcción de estructuras metálicas, Prestación de servicios y montaje industrial; y Mecanizado. Se trabajó con la cadena de suministro acotada, que tiene en cuenta solo los proveedores y clientes inmediatos.

En una segunda instancia se identificaron los procesos de segundo nivel. Allí se observó que todos los subgrupos trabajan con productos a diseño. El único que presenta una variación es el de "fabricación de aberturas de aluminio", que también realiza productos a pedido y para stock.

Seguidamente se definieron los indicadores y mejores prácticas asociados a los procesos identificados previamente con el fin de brindar elementos de soporte para recopilar información certera y definir donde se debe comenzar a efectuar cambios.

Este trabajo no presenta evidencia de la efectividad de las mejores prácticas recomendadas, pero se anticipa que la mayor resistencia se puede presentar al momento de implementar el uso de los indicadores planteados dada la cultura que caracteriza a la muestra en estudios respecto a falta de registros y manejo informal de la información.

Por tanto, se hace necesario continuar con nuevos estudios para confirmar que el uso de las mejores prácticas recomendadas modifica el desempeño de la cadena de suministro. Para ello se debería profundizar con un diagnóstico cuantitativo la implementación de dichas prácticas y por medio del seguimiento de los indicadores planteados realizar la medición de desempeño.

\section{Financiación}

Artículo de investigación derivado del proyecto de in vestigación titulado "Cadena de valor e impacto de las actividades clave en la conducta competitiva de las PyMES” de la Universidad Nacional del Centro de La Provincia de Buenos Aires. Fecha de inicio: Enero de 2012, Fecha de finalización: Diciembre de 2015.

\section{REFERENCIAS}

[1] S. Li, B. Ragu-Nathan, T. S. Ragu-Nathan, and S. S. Rao, "The impact of supply chain management practices on com petitive advantage and organizational performance", Omega, vol. 34, n 2 , pp. 107-124, 2006.

[2] K. C. Tan, V.R. Kannan, R. B. Handfield, and S. Ghosh, "Supply chain management: An empirical study of its impact on performance", International Journal of Operations \& Production Management, vol. 19, n 9/10, pp. 1034-1052, 1999

[3] A. Díaz Curbelo y F. Marrero Delgado, "El modelo SCOR y el Balance Scorecard: una poderosa combinación intangible para la gestión empresarial", Revista Científica Visión de Futuro, vol. 18, n 1, pp. 36-57, 2014.

[4] D. Lambert and T. Pohlen, "Supply chain metrics", International Journal of Logistics Management, vol. 12, $\mathrm{n}^{\circ} 1,2001$

[5] SCOR. Supply Chain Operations Reference Model 9.0, Supply Chain Council Inc., Estados Unidos, 2008.

[6] Ministerio de Industria de la Nación, La década ganada, 2003-2013. PyMEs: Protagonistas por dinámica, innovación y creatividad. Argentina, 2014. [Online]. Available: http://www.industria.gob.ar/wp-content/themes/twentyten/images/decada-ganada/PYMES-Protagonistas.pdf

[7] Ministerio de Producción, Ciencia y Tecnología de la Provincia de Buenos Aires, Plan Estratégico Productivo, Buenos Aires 2020. Argentina, 2012. [Online]. Available: http://www.mp.gba.gov.ar/downloads/PEPBA2020.pdf

[8] M. Kulfas, Las PyMEs argentinas en el escenario de post convertibilidad. Políticas públicas, situación y perspectivas. Comisión Económica para América Latina y el Caribe Cepal. Argentina, 2008. [Online]. Available: http://archivo. cepal.org/pdfs/2009/S2009508.pdf

[9] W. Sarache-Castro, D. Cárdenas-Aguirre y J. Giraldo, "Procedimiento para la definición y jerarquización de prioridades competitivas de fabricación. Aplicaciones en las PyMEs de la industria metalmecánica", Ingeniería y Competitividad, vol. $7, \mathrm{n}^{\circ} 2$, pp. 84-91, 2005 .

[10] Fundación Observatorio Pyme, La coyuntura de las PyMEs industriales. Argentina, 2013. [Online]. Available: http://www.observatoriopyme.org.ar/newsite/wp- content/ uploads/2014/09/FOP_IC_1305_Encuesta-Coyuntural-Primer-trimestre-de-2013-Industria.pdf

[11] Unión Industrial Argentina, Estudio de debilidades y desafíos tecnológicos del sector productivo. Argentina, 2008. [Online]. Available: http://www.uia.org.ar/departamento. do? nid $=682 \&$ id $=2$

[12] Subsecretaría de Indicadores Locales. "Segundo censo in dustrial de la ciudad de Olavarría. Informe Censo Indus trial 2011", Agencia de Desarrollo Local (ADELO), Argentina, 2011

[13] C. Rohvein, "Principios que delimitan el camino. sistema de gestión de calidad en PyMEs metalmecánicas", en II Encuentro Regional Argentino-Brasileño de Investigación Operativa. UNCPBA, Tandil, 2010.

[14] D. Paravié, G. Corres, S. Urrutia, C. Rohvein, and M. Jaureguiberry, "Las PyMEs de Olavarría, ¿se gestionan reactiva o proactivamente?", en II Encuentro Regional ArgentinoBrasileño de Investigación Operativa. UNCPBA, Tandil, 2010.

[15] Fundación Observatorio Pyme Regional Centro de la provincia de Buenos Aires, Industria manufacturera año 2008. Argentina, 2008. [Online]. Available: www.pymeregionales.org.ar/tandil/files/inf_publicacion_tandil_2008. pdf

[16] G. Roark, S. Urrutia, M. Jaureguiberry y D. Paravié, "Actividades predominantes a nivel nacional, regional y local de las PyMEs industriales Argentinas", Ciencias Administrativas, vol. 1, $\mathrm{n}^{\circ} 1$, pp. 1-16, 2013.

[17] D. Paravié, C. Rohvein, S. Urrutia, G. Roark y D. Ottogalli, "Diseño de un instrumento para evaluar el desempeño de las actividades que integran la cadena de valor de PyMEs metalmecánicas de Olavarría”, INGE CUC, vol. 8, n 1, pp. 7-32. 2012. 